\title{
Ginzburg-Landau Expansion in a Toy Model of Superconductor with Pseudogap
}

\author{
A.I.Posazhennikova, M.V.Sadovskii \\ Institute for Electrophysics, \\ Russian Academy of Sciences, Ural Branch, \\ Ekaterinburg, 620049, Russia \\ E-mail: posazhen@ief.uran.ru,sadovski@ief.uran.ru
}

JETP115, No.2 (1999)

cond-mat/9806199

\begin{abstract}
We propose a toy model of electronic spectrum of two-dimensional system with "hot-patches" on the Fermi surface, which leads to essential renormalization of spectral density (pseudogap). Within this model we derive GinzburgLandau expansion for both $s$-wave and $d$-wave Cooper pairing and analyze the influence of pseudogap formation on the basic properties of superconductors.
\end{abstract}

PACS numbers: 74.20.Fg, 74.20.De

Typeset using REVTEX 


\section{INTRODUCTION}

Among the number of anomalies of electronic properties of high-temperature superconductors particularly interesting is the observation of the pseudogap in the spectrum of elementary excitations in the underdoped region of the phase diagram [1.2]. Especially striking evidence of this unusual state were obtained in angle-resolved photoemission experiments on $B S C O$ [3, 4], which demonstrated the presence of significantly anisotropic changes in carrier spectral density in normal phase $\left(T>T_{c}\right)$. In these experiments the maximal value of the pseudogap was observed close to the point $(\pi, 0)$ of the Brillouin zone, while in the diagonal direction pseudogap changes were absent. Accordingly, in the vicinity of the point $(\pi, 0)$ the Fermi surface is practically completely destroyed, while it is conserved in the region around the zone diagonal. This picture is usually described as " $d$-wave" symmetry of the pseudogap, which is the same as the symmetry of superconducting gap in these systems. These anomalies persist up to the temperatures $T \simeq T^{*}$ which are much larger than $T_{c}$.

There is a number of theoretical approaches aiming to explain these anomalies. Among these, two main directions of thought can be described as the picture of Cooper pairs formation at temperatures much higher than $T_{c}$ [1, 1,5],6], and alternative scheme assuming the decisive role of fluctuations of antiferromagnetic short-range order [7-11].

Most of theoretical efforts up to now were dedicated to the studies of pseudogap state of HTSC in normal phase, i.e. for $T>T_{c}$. The aim of the present work is qualitative study of the influence of the pseudogap in electronic spectrum on the basic superconducting properties. We assume the validity of the ideology of antiferromagnetic fluctuations [7 [1], but introduce a very simplified model of the pseudogap state in normal phase, which allows the complete analysis in analytic form. Within this model we shall perform microscopic derivation of Ginzburg-Landau expansion for systems with both $s$-wave and $d$-wave pairing and analyze the qualitative effects of pseudogap formation (destruction of the parts of the Fermi surface) on basic superconducting properties.

\section{ELEMENTARY MODEL FOR A PSEUDOGAP STATE OF TWO-DIMENSIONAL ELECTRONIC SYSTEM.}

As mentioned above, we propose a greatly simplified model of the pseudogap state based upon the picture of well developed fluctuations of antiferromagnetic short-range order, which is close to the model of "hot-spots" on the Fermi surface 10,11. Let us assume that the Fermi surface of two-dimensional electronic system has the form shown in Fig.1. Analogous form of the Fermi surface was proposed in Ref. [12], where it was stressed that this form is very similar to that observed in a number of HTSC-systems [13,14]. We shall assume also that fluctuations of short-range order are static and Gaussian and define the appropriate correlation function as (Cf.Ref. [7]):

$$
S(\mathbf{q})=\frac{1}{\pi^{2}} \frac{\xi^{-1}}{\left(q_{x}-Q_{x}\right)^{2}+\xi^{-2}} \frac{\xi^{-1}}{\left(q_{y}-Q_{y}\right)^{2}+\xi^{-2}}
$$

where $\xi$-is the correlation length of these fluctuations. Here we take either $Q_{x}= \pm 2 k_{F}$ and $Q_{y}=0$ or $Q_{y}= \pm 2 k_{F}$ and $Q_{x}=0$. We shall assume that only electrons from flat "hot" 
patches on the Fermi surface shown in Fig.1 interact with these fluctuations. Effective interaction of electrons with these fluctuations will be described as $(2 \pi)^{2} W^{2} S(\mathbf{q})$, where the parameter $W$ has the dimension of energy and defines the characteristic energy scale (width) of the pseudogap. I It is clear that in our model this scattering is of purely one-dimensional nature. The choice of scattering vector $\mathbf{Q}=\left( \pm 2 k_{F}, 0\right)$ or $\mathbf{Q}=\left(0, \pm 2 k_{F}\right)$ corresponds in general to the picture of incommensurate fluctuations. Below we shall also consider a special case of commensurate scattering with $\mathbf{Q}=\left(\frac{\pi}{a}, \frac{\pi}{a}\right)$ (where $a$ is lattice spacing). In the limit of $\xi \rightarrow \infty$ our model can be solved exactly using methods proposed rather long ago in Refs. [15, 16, while in case of finite $\xi$ (although with some caution [10,11, 19]) by the method of Refs. 17, 18]. Below we shall only consider the toy case of $\xi \rightarrow \infty$, when the effective interaction with fluctuations (1) takes the following oversimplified form:

$$
(2 \pi)^{2} W^{2}\left\{\delta\left(q_{x} \pm 2 k_{F}\right) \delta\left(q_{y}\right)+\delta\left(q_{y} \pm 2 k_{F}\right) \delta\left(q_{x}\right)\right\}
$$

In this case it is possible to make the complete summation of all perturbation series for the electron scattered by these fluctuations [15, 16] and obtain the one-electron Green's function in the following form:

$$
G\left(\epsilon_{n}, p\right)=\int_{0}^{\infty} d \zeta e^{-\zeta} \frac{i \epsilon_{n}+\xi_{p}}{\left(i \epsilon_{n}\right)^{2}-\xi_{p}^{2}-\zeta W(\phi)^{2}}
$$

where $\xi_{p}=v_{F}\left(|\mathbf{p}|-p_{F}\right)\left(v_{F^{-}}\right.$Fermi velocity $), \epsilon_{n}=(2 n+1) \pi T$, while $W(\phi)$ is defined for $0 \leq \phi \leq \frac{\pi}{2}$ as:

$$
W(\phi)= \begin{cases}W & , 0 \leq \phi \leq \alpha, \frac{\pi}{2}-\alpha \leq \phi \leq \frac{\pi}{2} \\ 0 & , \alpha \leq \phi \leq \frac{\pi}{2}-\alpha\end{cases}
$$

where $\alpha=\operatorname{arctg}\left(\frac{p_{y}^{0}}{k_{F}}\right), \phi$ - polar angle, defining the direction of $\mathbf{p}$ in $\left(p_{x}, p_{y}\right)$ plane. For other values of $\phi$ we can define $W(\phi)$ analogously to (4) from the obvious symmetry. It is easily seen that changing $\alpha$ within the interval $0 \leq \alpha \leq \frac{\pi}{4}$, we in fact change the size of "hot patches" on the Fermi surface, where the "nesting" condition $\xi_{p-Q}=-\xi_{p}$ is satisfied. In particular, $\alpha=\pi / 4$ corresponds to the square Fermi surface, where the "nesting" condition is satisfied everywhere. Outside the "hot patches" (second inequality in (4)) the Green's function (3) is simply a free electron one.

Spectral density corresponding to Green's function (3) is given by:

$$
\begin{aligned}
& \rho\left(\epsilon \xi_{p}\right)=-\frac{1}{\pi} \operatorname{sign} \epsilon \operatorname{Im} G\left(\epsilon \xi_{p}\right)= \\
& \begin{cases}\frac{1}{W^{2}}\left(|\epsilon|+\xi_{p} \operatorname{sign} \epsilon\right) \theta\left(\epsilon^{2}-\xi_{p}^{2}\right) \exp \frac{\epsilon^{2}-\xi_{p}^{2}}{W^{2}} & , \text { for } 0 \leq \phi \leq \alpha, \frac{\pi}{2}-\alpha \leq \phi \leq \frac{\pi}{2} \\
\delta\left(\epsilon-\xi_{p}\right) & , \text { for } \alpha \leq \phi \leq \frac{\pi}{2}-\alpha\end{cases}
\end{aligned}
$$

\footnotetext{
${ }^{1}$ More formally we introduce here an effective interaction "constant" of electrons with fluctuations: $W_{\mathbf{p}}=W\left[\theta\left(p_{x}^{0}-p_{x}\right) \theta\left(p_{x}^{0}+p_{x}\right)+\theta\left(p_{y}^{0}-p_{y}\right) \theta\left(p_{y}^{0}+p_{y}\right)\right]$.
} 
and similarly for the other quadrants of the Brillouin zone. This expression (6) demonstrates non-Fermi-liquid (pseudogap) behavior with " $d$-wave" symmetry on the "hot patches" and free-like Fermi-liquid behavior on "cold patches" of the Fermi surface. Graphically the behavior of the spectral density on the "hot patch" is shown in Fig.2. Taking into account that the integral over the polar angle $\phi$ of the arbitrary function of $f(W(\phi))$ (where $W(\phi)$ is defined in (4)) is obviously given by:

$$
\int_{0}^{2 \pi} d \phi f(W(\phi))=8 \alpha f(W(\phi))+(2 \pi-8 \alpha) f(0),
$$

we can easily find from (6) the density of states:

$$
\frac{N(E)}{N_{0}(0)}=-\frac{1}{\pi} \int_{0}^{2 \pi} \frac{d \phi}{2 \pi} \int_{\infty}^{\infty} d \xi_{p} \operatorname{Im} G^{R}\left(\epsilon \xi_{p}\right)=4 \alpha / \pi N_{W}(\epsilon)+(1-4 \alpha / \pi) N_{0}(0)
$$

where $N_{0}(0)$ - is the density of states of free electrons at the Fermi level, while $N_{W}(\epsilon)$ - is the density of states of the one-dimensional problem (square Fermi surface), which was derived earlier in Refs. 15, 16]:

$$
\frac{N_{W}(\epsilon)}{N_{0}(\epsilon)}=\left|\frac{\epsilon}{W}\right| \int_{0}^{\frac{\epsilon^{2}}{W^{2}}} d \zeta \frac{e^{-\zeta}}{\sqrt{\frac{\epsilon^{2}}{W^{2}}-\zeta}}=2\left|\frac{\epsilon}{W}\right| \exp \left(-\frac{\epsilon^{2}}{W^{2}}\right) \operatorname{Erfi}\left(\frac{\epsilon}{W}\right)
$$

where $\operatorname{Erfi}(x)$ - is probability integral of imaginary argument.

In Fig.3 we show the graphic dependencies of the density of states in our model for different values of parameter $\alpha$, i.e. for "hot patches" of different sizes. It is seen that the pseudogap in the density of states is rather rapidly smeared with diminishing size of the "hot patches" and in general is not very pronounced. In some sense the effect of diminishing $\alpha$ is similar to that of diminishing correlation length of fluctuations $\xi$ [17, 18], so that our approximation of $\xi \rightarrow \infty$, is probably not a great limitation of our model. The advantage of this approximation on the other hand is in possibility to obtain all the major results in analytic form.

In conclusion of this section we present a short discussion of the case of commensurate fluctuations with $\mathbf{Q}=\left(\frac{\pi}{a}, \frac{\pi}{a}\right)$. In Fig.4 we show the model Fermi surface used in this case. The pseudogap is opened in the direction of diagonals in the Brillouin zone, which contradicts experiments on HTSC-systems, but this case is interesting from purely theoretical point. The model can be solved analogously to the previous case and generalizes the one-dimensional solution first found in Ref. [19]. One-electron Green's function has the form similar to (3), with $W(\phi)$ again a function of $\phi$ with period $\pi / 2$, but "rotated" with respect to the previous case by an angle of $\pi / 4$. For the interval of $-\pi / 4+\alpha \leq \phi \leq \pi / 4+\alpha$ we have:

$$
W(\phi)=\left\{\begin{array}{l}
W, \pi / 4-\alpha \leq \phi \leq \pi / 4+\alpha \\
0 \quad,-\pi / 4+\alpha \leq \phi \leq \pi / 4-\alpha
\end{array}\right.
$$

where $0 \leq \alpha \leq \pi / 4$. Also in this case we must take into account a different combinatorics of Feynman diagrams, corresponding to the scattering of electrons on commensurate fluctuations [19]. As a result in Eq.(3) we have to replace the integral 


$$
\int_{0}^{\infty} d \zeta e^{-\zeta}
$$

by

$$
\int_{0}^{\infty} d \zeta \frac{1}{2 \sqrt{\pi \zeta}} e^{-\zeta / 4}
$$

\section{EQUATION FOR $T_{C}$.}

Let us now consider the case of superconducting pairing in our model. Assume the usual separable form of pairing interaction [20]:

$$
V\left(\mathbf{p}, \mathbf{p}^{\prime}\right)=V\left(\phi, \phi^{\prime}\right)=-V e(\phi) e\left(\phi^{\prime}\right)
$$

where $\phi$ - is again an angle, determining the direction of electronic momentum $\mathbf{p}$ in the plane, and for $e(\phi)$ we assume the simple model dependence:

$$
e(\phi)=\left\{\begin{array}{ll}
1 & ,(s \text {-wave pairing }) \\
\sqrt{2} \cos (2 \phi) & ,(d \text {-wave pairing })
\end{array} .\right.
$$

Interaction constant of attractive interaction $V$ is assumed as usual to be non-zero in some region of the width of $2 \omega_{c}$ around the Fermi level $\left(\omega_{c}\right.$ - is characteristic frequency of quanta, responsible for attractive interaction). In this case the superconducting gap (order parameter) takes the following form:

$$
\Delta(\mathbf{p}) \equiv \Delta(\phi)=\Delta e(\phi)
$$

Equation for superconducting transition temperature $T_{c}$ is obtained from the usual equation determining Cooper's instability:

$$
1-\chi(0,0)=0
$$

where the generalized Cooper's susceptibility $\chi(0,0)$ can be calculated by exact summation of all diagrams, taking into account the scattering on fluctuations of short-range order (2), in a way similar to that used in Refs. [15, 16] to calculate the polarization operator. As a result we obtain the following equation for $T_{c}$ :

$$
\begin{array}{r}
\frac{1}{V}=-\int_{0}^{\infty} d \zeta e^{-\zeta} T_{c} \sum_{n} \int_{0}^{\infty} \frac{d^{2} p}{(2 \pi)^{2}} e^{2}(\phi)\left\{\left(G_{\zeta W^{2}}\left(\epsilon_{n} ; \mathbf{p}, \mathbf{p}\right) G_{\zeta W^{2}}\left(-\epsilon_{n} ;-\mathbf{p},-\mathbf{p}\right)+\right.\right. \\
F_{\zeta W^{2}}\left(\epsilon_{n} ; \mathbf{p}, \mathbf{p}-\mathbf{Q}\right) F_{\zeta W^{2}}\left(-\epsilon_{n} ;-\mathbf{p},-\mathbf{p}+\mathbf{Q}\right\},
\end{array}
$$

where

$$
\begin{aligned}
G_{\zeta W^{2}}\left(\epsilon_{n} ; \mathbf{p}, \mathbf{p}\right) & =\frac{i \epsilon_{n}+\xi_{p}}{\left(i \epsilon_{n}\right)^{2}-\xi_{p}^{2}-\zeta W^{2}(\phi)} \\
F_{\zeta W^{2}}\left(\epsilon_{n} ; \mathbf{p}, \mathbf{p}-\mathbf{Q}\right) & =\frac{\sqrt{\zeta} W(\phi)}{\left(i \epsilon_{n}\right)^{2}-\xi_{p}^{2}-\zeta W^{2}(\phi)}
\end{aligned}
$$


are appropriately defined "normal" and "anomalous" Green's functions for the system with dielectric gap 15, 16].

After some standard transformations from (18) we get:

$$
\frac{1}{V}=\int_{0}^{\infty} d \zeta e^{-\zeta} T_{c} \sum_{n} \int_{0}^{\infty} \frac{d^{2} p}{(2 \pi)^{2}} \frac{e^{2}(\phi)}{\epsilon_{n}^{2}+\xi_{p}^{2}+\zeta W(\phi)^{2}}
$$

so that after summation over frequencies we obtain:

$$
\frac{1}{V}=\frac{N(0)}{2 \pi} \int_{0}^{\infty} d \zeta e^{-\zeta} \int_{-\infty}^{\infty} d \xi \int_{0}^{2 \pi} d \phi \frac{e^{2}(\phi)}{2 \sqrt{\xi^{2}+\zeta W(\phi)^{2}}} \operatorname{th} \frac{\sqrt{\xi^{2}+\zeta W(\phi)^{2}}}{2 T_{c}} .
$$

Performing the angular integration over $\phi$ similarly to (7), for the case of $s$-wave pairing we get:

$$
\frac{1}{g}=\frac{4 \alpha}{\pi} \int_{0}^{\infty} d \zeta e^{-\zeta} \int_{0}^{\omega_{c}} d \xi \frac{1}{\sqrt{\xi^{2}+\zeta W^{2}}} t h \frac{\left.\sqrt{\xi^{2}+\zeta W^{2}}\right)}{2 T_{c}}+\left(1-\frac{4 \alpha}{\pi}\right) \int_{0}^{\omega_{c}} d \xi \frac{1}{\xi} t h \frac{\xi}{2 T_{c}} .
$$

while for the case of $d$-wave pairing:

$$
\begin{array}{rl}
\frac{1}{g}=\frac{\sin (4 \alpha)+4 \alpha}{2 \pi} \int_{0}^{\infty} d \zeta e^{-\zeta} \int_{0}^{\omega_{c}} & d \xi \frac{1}{\sqrt{\xi^{2}+\zeta W^{2}}} \operatorname{th} \frac{\left.\sqrt{\xi^{2}+\zeta W^{2}}\right)}{2 T_{c}}+ \\
& \frac{\pi-4 \alpha-\sin (4 \alpha)}{2 \pi} \int_{0}^{\omega_{c}} d \xi \frac{1}{\xi} \operatorname{th} \frac{\xi}{2 T_{c}} .
\end{array}
$$

where $g=N(0) V$ - is dimensionless pairing coupling constant. In Fig.5(a,b) we show the graphic dependencies of $T_{c} / T_{c 0}$ on the parameter $W / T_{c 0}$, determining the effective width of the pseudogap, for different values of $\alpha\left(T_{c 0}\right.$-is superconducting transition temperature for an "ideal" system without pseudogap). It is clearly seen that for both types of pairing the appearance of the pseudogap on "hot patches" of the Fermi surface leads to significant suppression of $T_{c}$, which becomes stronger with the growing size of "hot patches". Naturally enough for the case of $d$ - wave pairing this effect of $T_{c}$ suppression is much stronger than in $s$ - wave case, because the dielectrization of electronic spectrum (pseudogap) operates "out of phase" with pairing interaction.

In case of commensurate fluctuations (Fig.4) and $d$-wave pairing the $T_{c}$ - equation takes the following form:

$$
\begin{array}{rl}
\frac{1}{g}=\frac{4 \alpha-\sin (4 \alpha)}{2 \pi} \int_{0}^{\infty} d \zeta \frac{1}{2 \sqrt{\pi \zeta}} e^{-\zeta / 4} \int_{0}^{\omega_{c}} & d \xi \frac{1}{\sqrt{\xi^{2}+\zeta W^{2}}} \operatorname{th} \frac{\left.\sqrt{\xi^{2}+\zeta W^{2}}\right)}{2 T_{c}}+ \\
& \frac{\pi-4 \alpha+\sin (4 \alpha)}{2 \pi} \int_{0}^{\omega_{c}} d \xi \frac{1}{\xi} \operatorname{th} \frac{\xi}{2 T_{c}}
\end{array}
$$

The appropriate dependencies of $\frac{T_{c}}{T_{c 0}}$ on $\frac{W}{T_{c} 0}$ for different values of $\alpha$ in this case are shown in Fig.6. Here the effect of $T_{c}$ suppression by the pseudogap is less significant because the maximum value of superconducting gap is achieved on "cold patches" of the Fermi surface, where pseudogap is absent. 


\section{GINZBURG-LANDAU EXPANSION.}

Ginzburg-Landau expansion for the difference of free energy density of superconducting and normal state can be written in usual form:

$$
F_{s}-F_{n}=A\left|\Delta_{q}\right|^{2}+q^{2} C\left|\Delta_{q}\right|^{2}+\frac{B}{2}\left|\Delta_{q}\right|^{4}
$$

where $\Delta_{q}$ - is the amplitude of the Fourier component of the order parameter:

$$
\Delta(\phi, q)=\Delta_{q} e(\phi)
$$

Actually (24) is determined by the loop expansion for free energy of electron scattered by fluctuations of the order parameter with small wave-vector q, which is shown in Fig.7, where in all loops we have to perform exact summation of all scattering processes due to fluctuations of short range order (2). Again this is easily done by methods of Refs. 15, 16. In other respects all calculations are similar to that done by us in Ref. [20]. 2] As in this previous work the subtraction of the second diagram in Fig.7 guarantees the zero value of the coefficient $A$ at the transition point $T=T_{c}$. Finally, the coefficients of Ginzburg-Landau expansion are written as:

$$
A=A_{0} K_{A} ; \quad C=C_{0} K_{C} ; \quad B=B_{0} K_{B},
$$

where $A_{0}, C_{0}$ and $B_{0}$ define these coefficients for the case of two-dimensional isotropic $s$-wave superconductor in the absence of pseudogap $(\alpha=0)$ :

$$
A_{0}=N(0) \frac{T-T_{c}}{T_{c}} ; \quad C_{0}=N(0) \frac{7 \zeta(3)}{32 \pi^{2}} \frac{v_{F}^{2}}{T_{c}^{2}} ; \quad B_{0}=N(0) \frac{7 \zeta(3)}{8 \pi^{2} T_{c}^{2}}
$$

while all peculiar dependencies characteristic to our model are contained in dimensionless coefficients $K_{A}, K_{C}$ and $K_{B}$. In the absence of the pseudogap all these dimensionless coefficients are equal to 1 , only in the case of $D$-wave pairing $K_{B}=3 / 2$.

Direct calculations give:

$$
A=N(0) \frac{T-T_{c}}{2 T_{c}^{2}} \frac{1}{2 \pi} \int_{0}^{\infty} d \zeta e^{-\zeta} \int_{0}^{\omega_{c}} d \xi \int_{0}^{2 \pi} d \phi \frac{e^{2}(\phi)}{c h^{2} \frac{\sqrt{\xi^{2}+\zeta W(\phi)^{2}}}{2 T_{c}}}
$$

so that after the integration over $\phi$ we obtain:

$$
K_{A}=\frac{1}{2 T_{c}} \beta_{a} \int_{0}^{\infty} d \zeta e^{-\zeta} \int_{0}^{\omega_{c}} d \xi \frac{1}{\operatorname{ch}^{2} \frac{\sqrt{\xi^{2}+\zeta W^{2}}}{2 T_{c}}}+1-\beta_{a}
$$

where

\footnotetext{
${ }^{2}$ Similar in spirit Ginzburg-Landau analysis for Peierls transition in quais-one-dimensinal systems was performed in Ref. [21.
} 


$$
\beta_{a}= \begin{cases}\frac{4 \alpha}{\pi} & , s \text {-wave pairing } \\ \frac{4 \alpha+\sin (4 \alpha)}{\pi} & , d \text {-wave pairing. }\end{cases}
$$

In Fig.8 we show the dependencies of $K_{A}$ on the pseudogap width $W / T_{c 0}$ for different values of $\alpha$. We show these only for $s$-wave case, for $d$-wave pairing these dependencies are qualitatively similar, but all the changes of the coefficient take place on much smaller scales of $W / T_{c 0}$, as in Fig.5.

To calculate $C$ it is necessary to make Taylor expansion in powers of $\mathbf{q}$ in

$$
\begin{array}{r}
-\int_{0}^{\infty} d \zeta e^{-\zeta} T_{c} \sum_{n} \int_{0}^{\infty} \frac{d^{2} p}{(2 \pi)^{2}} e^{2}(\phi)\left\{\left(G_{\zeta W(\phi)^{2}}\left(\epsilon_{n} ; \mathbf{p}_{+}, \mathbf{p}_{+}\right) G_{\zeta W(\phi)^{2}}\left(-\epsilon_{n} ;-\mathbf{p}_{-},-\mathbf{p}_{-}\right)+\right.\right. \\
F_{\zeta W(\phi)^{2}}\left(\epsilon_{n} ; \mathbf{p}_{+}, \mathbf{p}_{+}-\mathbf{Q}\right) F_{\zeta W(\phi)^{2}}\left(-\epsilon_{n} ;-\mathbf{p}_{-},-\mathbf{p}_{-}+\mathbf{Q}\right\}
\end{array}
$$

where $\mathbf{p}_{ \pm}=\mathbf{p} \pm \frac{\mathbf{q}}{2}$, and find the coefficient of $\mathbf{q}^{\mathbf{2}}$. To simplify expressions let us introduce the following notations: $G_{\zeta W(\phi)^{2}}\left(\epsilon_{n} ; \mathbf{p}, \mathbf{p}\right) \equiv G_{p p} ; F_{\zeta W(\phi)^{2}}\left(\epsilon_{n} ; \mathbf{p}, \mathbf{p}-\mathbf{Q}\right) \equiv F_{p p-Q}$.

After some complicated but purely technical calculations we obtain $C$ in the following form:

$$
C=-T_{c} \frac{N(0)}{2 \pi} v_{F}^{2} \sum_{n} \int_{0}^{\infty} d \zeta e^{-\zeta} \int d \xi \int_{0}^{2 \pi} d \phi \frac{\cos (\phi)^{2} e^{2}(\phi)\left(\xi^{2}-3 \epsilon_{n}^{2}-3 \zeta W(\phi)^{2}\right)}{2\left(\epsilon_{n}^{2}+\xi^{2}+\zeta W(\phi)^{2}\right)^{3}} .
$$

Accordingly, after the integration over $\xi$ and $\phi$ we get for the dimensionless coefficient $K_{C}$ :

$$
K_{C}=\beta_{c} \frac{4 \pi^{3} T_{c}^{3}}{7 \zeta(3)} \int_{0}^{\infty} d \zeta e^{-\zeta} \sum_{n} \frac{1}{\left(\sqrt{\epsilon_{n}^{2}+\zeta W^{2}}\right)^{3}}+1-\beta_{c}
$$

where $\beta_{c}=\beta_{a}$ (Cf.(30)). Appropriate dependencies of $K_{C}$ on $W / T_{c 0}$ for the case of $s$-wave pairing are shown in Fig.9. In case of $d$-wave pairing the picture is similar, but again all the changes of the coefficient $K_{C}$ take place on smaller scales of $W / T_{c 0}$.

Calculation of the fourth order term in Ginzburg-Landau expansion is technically much more complicated. To obtain the expression for the coefficient $B$ we have to calculate the trace of the product of four Green's functions $\hat{\mathbf{G}}_{\mathbf{p}}$ which are Nambu matrices of normal and anomalous Green' functions (19):

$$
\hat{\mathbf{G}}_{\mathbf{p}}=\left(\begin{array}{cc}
G_{p p} & F_{p p-Q} \\
F_{p-Q p} & G_{p-Q p-Q}
\end{array}\right) .
$$

After performing the trace of $\hat{\mathbf{G}_{\mathbf{p}}} \widehat{\mathbf{G}_{-\mathbf{p}}} \hat{\mathbf{G}_{\mathbf{p}} \mathbf{G}_{-\mathbf{p}}}$ the coefficient $B$ takes the form:

$$
\begin{array}{r}
B=N(0) T_{c} \sum_{\epsilon_{n}} \int_{0}^{\infty} d \zeta e^{-\zeta} \int_{0}^{\infty} \frac{d^{2} p}{(2 \pi)^{2}} e^{4}(\phi)\left\{\left(G_{p, p} G_{-p,-p}+F_{p, p-Q} F_{-p,-p+Q}\right)^{2}+\right. \\
G_{p, p} G_{-p,-p} F_{-p+Q, p} F_{p-Q, p}+G_{-p+Q,-p+Q} G_{-p,-p} F_{p, p-Q} F_{p-Q, p}+ \\
\left.G_{p, p} G_{p-Q, p-Q} F_{-p+Q,-p} F_{-p,-p+Q}+G_{p-Q, p-Q} G_{-p+Q,-p+Q} F_{p, p-Q} F_{-p,-p+Q}\right\} .
\end{array}
$$


It can be seen by direct calculations that the sum of the last four terms in (34) gives zero, so that:

$$
B=N(0) T_{c} \sum_{\epsilon_{n}} \int_{0}^{\infty} d \zeta e^{-\zeta} \int_{0}^{\infty} \frac{d^{2} p}{(2 \pi)^{2}} e^{4}(\phi)\left(G_{p, p} G_{-p,-p}+F_{p, p-Q} F_{-p,-p+Q}\right)^{2}
$$

From here we obtain:

$$
B=\frac{N(0) T_{c}}{2 \pi} \sum_{n} \int_{0}^{\infty} d \zeta e^{-\zeta} \int_{-\infty}^{\infty} d \xi \int_{0}^{2 \pi} d \phi \frac{e^{4}(\phi)}{\left(\epsilon_{n}^{2}+\xi^{2}+\zeta W(\phi)^{2}\right)^{2}}
$$

and after the integration over $\xi$ and $\phi$ we obtain $K_{B}$ in the form similar to (33):

$$
K_{B}=\beta_{b} \frac{4 \pi^{3} T_{c}^{3}}{7 \zeta(3)} \int_{0}^{\infty} d \zeta e^{-\zeta} \sum_{n} \frac{1}{\left(\sqrt{\epsilon_{n}^{2}+\zeta W^{2}}\right)^{3}}+1-\beta_{b}
$$

where

$$
\beta_{b}=\left\{\begin{array}{ll}
\frac{4 \alpha}{\pi} & , s \text {-wave pairing } \\
\frac{4 \alpha}{\pi}+\frac{4 \sin (4 \alpha)}{3 \pi}+\frac{\sin (8 \alpha)}{6 \pi}, & , d \text {-wave pairing }
\end{array} .\right.
$$

Thus for the case of $s$-wave pairing coefficients $K_{B}$ and $K_{C}$ just coincide.

In conclusion of this section we shall give explicit expressions for dimensionless GinzburgLandau coefficients for the case of $d$-wave pairing in the model of commensurate fluctuations of short-range order:

$$
\begin{gathered}
K_{A}=\beta_{a} \frac{1}{2 T_{c}} \int_{0}^{\infty} d \zeta \frac{1}{2 \sqrt{\pi \zeta}} e^{-\zeta / 4} \int_{0}^{\omega_{c}} d \xi \frac{1}{c h^{2} \frac{\sqrt{\xi^{2}+\zeta W^{2}}}{2 T_{c}}}+1-\beta_{a} \\
K_{C, B}=\beta_{c, b} \frac{4 \pi^{3} T_{c}^{3}}{7 \zeta(3)} \int_{0}^{\infty} d \zeta \frac{1}{2 \sqrt{\pi \zeta}} e^{-\zeta / 4} \sum_{n} \frac{1}{\left(\sqrt{\epsilon_{n}^{2}+\zeta W^{2}}\right)^{3}}+1-\beta_{c, b},
\end{gathered}
$$

where

$$
\beta_{a}=\beta_{c}=\frac{4 \alpha-\sin (4 \alpha)}{\pi} ; \quad \beta_{b}=\frac{4 \alpha}{\pi}-\frac{\sin (4 \alpha)}{6 \pi}(5+\cos (4 \alpha)) .
$$

It is a simple task to give the appropriate dependencies of these coefficients on $W / T_{c 0}$

for different values of $\alpha$. Qualitatively these dependencies are more or less similar to that obtained in incommensurate case, while all the differences are mainly due to another (larger) scale on the $W / T_{c 0}$ axis(Cf. Fig.6).

\section{PHYSICAL PROPERTIES OF SUPERCONDUCTORS WITH PSEUDOGAP.}

It is well known that Ginzburg-Landau expansion defines two characteristic lengths of superconducting state: the coherence length and penetration depth of magnetic field. 
The coherence length for given temperature $\xi(T)$ determines characteristic scale of inhomogeneities of the order parameter $\Delta$, i.e. in fact the "size" of Cooper's pair:

$$
\xi^{2}(T)=-\frac{C}{A}
$$

In the usual case (in the absence of pseudogap):

$$
\begin{array}{r}
\xi_{B C S}^{2}(T)=-\frac{C_{0}}{A_{0}}, \\
\xi_{B C S}(T) \approx 0.74 \frac{\xi_{0}}{\sqrt{1-T / T_{c}}},
\end{array}
$$

where $\xi_{0}=0.18 v_{F} / T_{c}$. In our case:

$$
\frac{\xi^{2}(T)}{\xi_{B C S}^{2}(T)}=\frac{K_{C}}{K_{A}} .
$$

Appropriate dependencies of $\xi^{2}(T) / \xi_{B C S}^{2}(T)$ on $W / T_{c 0}$ for the case of $d$-wave pairing and incommensurate fluctuations of short range order are shown in Fig.10.

For the penetration depth in usual case we have:

$$
\lambda_{B C S}(T)=\frac{1}{\sqrt{2}} \frac{\lambda_{0}}{\sqrt{1-T / T_{c}}},
$$

where $\lambda_{0}^{2}=\frac{m c^{2}}{4 \pi n e^{2}}$ defines penetration depth for $T=0$. In general case we have the following expression for penetration depth via Ginzburg-Landau coefficients:

$$
\lambda^{2}(T)=-\frac{c^{2}}{32 \pi e^{2}} \frac{B}{A C}
$$

Thus for our model:

$$
\frac{\lambda(T)}{\lambda_{B C S}(T)}=\left(\frac{K_{B}}{K_{A} K_{C}}\right)^{1 / 2} .
$$

Graphic dependencies of this parameter on the effective width of the pseudogap for the case of $d$-wave pairing are shown in Fig.11.

Consider now Ginzburg-Landau parameter:

$$
\kappa=\frac{\lambda(T)}{\xi(T)}=\frac{c}{4 e C} \sqrt{B / 2 \pi} .
$$

In our model of superconductor:

$$
\frac{\kappa}{\kappa_{B C S}}=\frac{\sqrt{K_{B}}}{K_{C}}
$$

where 


$$
\kappa_{B C S}=\frac{3 c}{\sqrt{7 \zeta(3)} e} \frac{T_{c}}{v_{F}^{2} \sqrt{N(0)}}
$$

-is Ginzburg-Landau parameter for the usual case (without pseudogap). The dependencies of $\kappa / \kappa_{B C S}$ on $W / T_{c 0}$ for the case of $d$-wave pairing are shown in Fig. 12 .

Close to $T_{c}$ the upper critical magnetic field $H_{c 2}$ is defined by Ginzburg-Landau coefficients as:

$$
H_{c 2}=-\frac{\phi_{0}}{2 \pi} \frac{A}{C}
$$

where $\phi_{0}=c \pi / e-$ magnetic flux quantum. Then the slope of the upper critical field at $T_{c}$ is given by:

$$
\left|\frac{d H_{c 2}}{d T}\right|_{T_{c}}=\frac{24 \pi \phi_{0}}{7 \zeta(3) v_{F}^{2}} T_{c} \frac{K_{A}}{K_{C}} .
$$

Graphic dependencies of this slope $\left|\frac{d H_{c 2}}{d T}\right|_{T_{c}}$, normalized by the slope at $T_{c 0}$, on the effective width of the pseudogap $W / T_{c 0}$ for the case of $d$-wave pairing are shown in Fig.13. It is seen that the slope drops with the growth of the pseudogap.

We can also calculate the discontinuity of specific heat at superconducting transition, which is given by:

$$
\frac{C_{s}-C_{n}}{\Omega}=\frac{T_{c}}{B}\left(\frac{A}{T-T_{c}}\right)^{2},
$$

where $C_{s}, C_{n}$ - are specific heats of superconducting and normal states, $\Omega$ - is system volume. From here the specific heat discontinuity at $T_{c 0}(W=0)$ is:

$$
\left(\frac{C_{s}-C_{n}}{\Omega}\right)_{T_{c 0}}=N(0) \frac{8 \pi^{2} T_{c 0}}{7 \zeta(3)} .
$$

Then the specific heat discontinuity in our model can be expressed via dimensionless coefficients $K_{A}$ and $K_{B}$ as:

$$
\frac{\left(C_{s}-C_{n}\right)_{T_{c}}}{\left(C_{s}-C_{n}\right)_{T_{c 0}}}=\frac{T_{c}}{T_{c 0}} \frac{K_{A}^{2}}{K_{B}} .
$$

The appropriate dependencies on the effective width of the pseudogap for the case of $d$ wave pairing are shown in Fig.14. It is seen that specific heat discontinuity is significantly suppressed with the growth of the pseudogap.

Analogous dependencies of all these physical properties for the case of $s$-wave pairing, and also for the model of commensurate fluctuations, qualitatively are more or less similar to that shown in Figs.10-14, the only major difference is in the scale of $W / T_{c 0}$ as in Figs.5-6. 


\section{CONCLUSION.}

In this paper we have analyzed an oversimplified (toy) model of the pseudogap in twodimensional electronic system, which is however in some respects corresponding to some of the observed anomalies of electronic structure of underdoped HTSC-systems. In particular, it is rather easy to obtain the " $d$-wave" symmetry of the pseudogap state, which is determined by the appropriate positions of "hot patches" on the Fermi surface, and strong scattering by fluctuations of short-range (e.g. antiferromagnetic) order. Obviously this model can be easily generalized to the case of larger number of "hot patches", so that it can be made more similar to the model of "hot spots" [10,11].

The main simplifying assumption as well as the main defect of this model is our use of rather unrealistic limit of $\xi \rightarrow \infty$ for the correlation length of fluctuations, which actually helps us to get all the main results in more or less analytic form. In real systems the value of $\xi$ is not very large and depends on temperature and doping, becoming an important independent parameter controlling the physical picture. In principle our model can be generalized for the case of finite $\xi$ along the lines of Refs. 17,18. However, all calculations in this case become much more complicated. At the same time it is clear enough that the effect of finite $\xi$ reduces mainly to the smearing of the pseudogap [17, 18 and in this sense is somehow modelled by diminishing the size of "hot patches" in our model. It is more or less correct for the effects determined mainly by the density of states (e.g. superconducting transition temperature $T_{c}$ ). At the same time this is hardly so in case of the physical properties, defined mainly by two-particle Green's function, such as the coefficient $C$ of the gradient term in Ginzburg-Landau expansion.

Another radical simplification of our model is an assumption of static (and Gaussian) nature of fluctuations of short range order. This assumption can be justified in the limit of high enough temperatures $T \gg \omega_{s f}$ (where $\omega_{s f}$ - is characteristic frequency of spin fluctuations) [1]. Accordingly the assumption of the static nature of these fluctuations is rather problematic at temperatures close to $T_{c}$. However, our analysis of Ginzburg-Landau expansion for different types of pairing is apparently a rather good description of the main effect of the "destruction" of certain parts of the Fermi surface on the main properties of a superconductor with the pseudogap. It demonstrates an important role of pseudogap anomalies in formation of superconducting phase for those part of the phase diagram of HTSC-systems, where pseudogap anomalies are observed already in normal state. Analysis of more realistic models is supposed to be done in future studies.

The authors are grateful to E.Z.Kuchinskii for useful discussions.

This work was performed with partial support from Russian Basic Research Foundation under the grant No.96-02-16065, as well as under the projects No.IX.1 of State Program "Statistical Physics" and No.96-051 of State Program on HTSC of the Russian Ministry of Science. 


\section{Figure Captions:}

Fig.1. Fermi surface of two-dimensional system. "Hot patches" are shown by thick lines, with width of the order of $\sim \xi^{-1}$.

Fig.2. Spectral density of Green's function on the "hot patch" of the Fermi surface.

$(1)-\xi_{p}=0 ;(2)-\xi_{p}=0.1 W ;(3)-\xi_{p}=0.5 W$.

Fig.3. Electronic density of states for "hot patches" of different sizes:

(1) $-\alpha=\pi / 4 ;(2)-\alpha=\pi / 6 ;(3)-\alpha=\pi / 8 ;(4)-\alpha=\pi / 12 ;(5)-\alpha=\pi / 24$.

Fig.4. Fermi surface in the Brillouin zone of two-dimensional system in "hor patches" model for the case of commensurate fluctuations of short range order, corresponding to period doubling. A new Brillouin zone appearing after the establishment of long range (e.g. antiferromagnetic) order is also shown.

Fig.5. $T_{c} / T_{c 0}$ dependence on the effective width of the pseudogap $W / T_{c 0}$ for "hot patches" of different sizes in the model of incommensurate fluctuations.

(a) $-s$-wave pairing: (1) $-\alpha=\pi / 4 ;(2)-\alpha=\pi / 6$; (3)- $\alpha=\pi / 8$; (4) $-\alpha=\pi / 12$.

(b) $-d$-wave pairing: (1) $-\alpha=\pi / 4$; (2) $-\alpha=\pi / 6$; (3) $-\alpha=\pi / 8$; (4) $-\alpha=\pi / 12$.

Fig.6. $T_{c} / T_{c 0}$ dependence on the effective width of the pseudogap $W / T_{c 0}$ for "hot patches" of different sizes in the model of commensurate fluctuations for the case of $d$-wave pairing:

(1) $-\alpha=\pi / 4 ;(2)-\alpha=\pi / 6 ;(3)-\alpha=\pi / 8 ;(4)-\alpha=\pi / 12$.

Fig.7. Diagrammatic representation of Ginzburg-Landau expansion in the field of fluctuations of short range order. Electronic lines represent Nambu matrices composed of normal and anomalous Green's functions (19). Loops are averaged over $\zeta$ with distribution (11) or (12). Second loop is calculated for $q=0$ and $T=T_{c}$.

Fig.8. Dependence of the coefficient $K_{A}$ on effective width of the pseudogap $W / T_{c 0}$ for "hot patches" of different sizes in the model of incommensurate fluctuations in the case of $s$-wave pairing:

(1) $-\alpha=\pi / 4 ;(2)-\alpha=\pi / 6 ;(3)-\alpha=\pi / 8 ;(4)-\alpha=\pi / 12$.

Fig.9. Dependence of coefficients $K_{B}, K_{C}$ on the effective width of the pseudogap $W / T_{c 0}$ for "hot patches" of different sizes in the model of incommensurate fluctuations in the case of $s$-wave pairing:

(1) $-\alpha=\pi / 4 ;(2)-\alpha=\pi / 6 ;(3)-\alpha=\pi / 8 ;(4)-\alpha=\pi / 12$.

Fig.10. Dependence of coherence length $\xi^{2}(T) / \xi_{B C S}^{2}(T)$ on the effective width of the pseudogap $W / T_{c 0}$ in case of $d$-wave pairing:

(1) $-\alpha=\pi / 4 ;(2)-\alpha=\pi / 8 ;(3)-\alpha=\pi / 12$.

Fig.11. Dependence of penetration depth $\lambda(T) / \lambda_{B C S}(T)$ on the effective width of the pseudogap $W / T_{c 0}$ in case of $d$-wave pairing:

(1) $-\alpha=\pi / 4 ;(2)-\alpha=\pi / 8 ;(3)-\alpha=\pi / 12$.

Fig.12. Dependence of Ginzburg-Landau parameter $\kappa / \kappa_{B C S}$ on the effective width of the pseudogap $W / T_{c 0}$ in case of $d$-wave pairing:

(1) $-\alpha=\pi / 4 ;(2)-\alpha=\pi / 8$; (3) $-\alpha=\pi / 12$.

Fig.13. Dependence of normalized slope of the upper critical field on the effective width of the pseudogap $W / T_{c 0}$ in case of $d$-wave pairing:

(1) $-\alpha=\pi / 4 ;(2)-\alpha=\pi / 8 ;(3)-\alpha=\pi / 12$.

Fig.14. Dependence of normalized discontinuity of specific heat on the effective width of the pseudogap $W / T_{c 0}$ in case of $d$-wave pairing:

(1) $-\alpha=\pi / 4 ;(2)-\alpha=\pi / 8 ;(3)-\alpha=\pi / 12$. 


\section{REFERENCES}

[1] M.Randeria. Varenna Lectures 1997, preprint cond-mat/9710223

[2] M.Randeria, J.C.Campuzano, Varenna Lectures 1997, preprint cond-mat/9709107

[3] H.Ding et al. Nature 382,51(1996)

[4] H.Ding et al. Phys.Rev.Lett. 78,2628(1997)

[5] V.B.Geshkenbein,L.B.Ioffe,A.I.Larkin. Phys.Rev. B55,3173(1997)

[6] V.Emery, S.A.Kivelson, O.Zachar. Phys.Rev. B56,6120(1997)

[7] A.P.Kampf,J.R.Schrieffer. Phys.Rev. B41,6399(1990), B42,7967(1990)

[8] V.Barzykin,D.Pines. Phys.Rev. B52,13585(1995)

[9] D.Pines. Tr.J. of Physics 20,535(1996)

[10] J.Schmalian, D.Pines, B.Stojkovic. Phys.Rev.Lett. 80, 3839(1998)

[11] J.Schmalian, D.Pines, B.Stojkovic. Preprint cond-mat/9804129.

[12] A.T.Zheleznyak, V.M.Yakovenko, I.E.Dzyaloshinskii. Phys.Rev. B55,3200(1997)

[13] D.S.Dessau et al. Phys.Rev.Lett. 71, 2781(1993)

[14] Z.X.Shen et al. Science 267, 343(1995)

[15] M.V.Sadovskii. Zh.Eksp.Teor.Fiz. 66, 1720(1974); Sov.Phys.-JETP 39, 845 (1974)

[16] M.V.Sadovskii. Fiz.Tverd.Tela 16, 2504(1974); Sov.Phys.-Solid State 16, 1632 (1974)

[17] M.V.Sadovskii. Zh.Eksp.Teor.Fiz. 77, 2070(1979); Sov.Phys.-JETP 50, 989 (1979)

[18] M.V.Sadovskii, A.A.Timofeev. Sverkhprovodimost' 4, 11(1991);

J. Moscow Phys. Soc. 1,391(1991)

[19] W.Wonneberger, R.Lautenschlager. J.Phys. C9,2865(1976)

[20] A.I.Posazhennikova, M.V.Sadovskii. Zh.Eksp.Teor.Fiz 112, 2124 (1997); JETP 85, 2124 (1997)

[21] R.H.McKenzie. Phys.Rev. B52, 16428 (1995) 


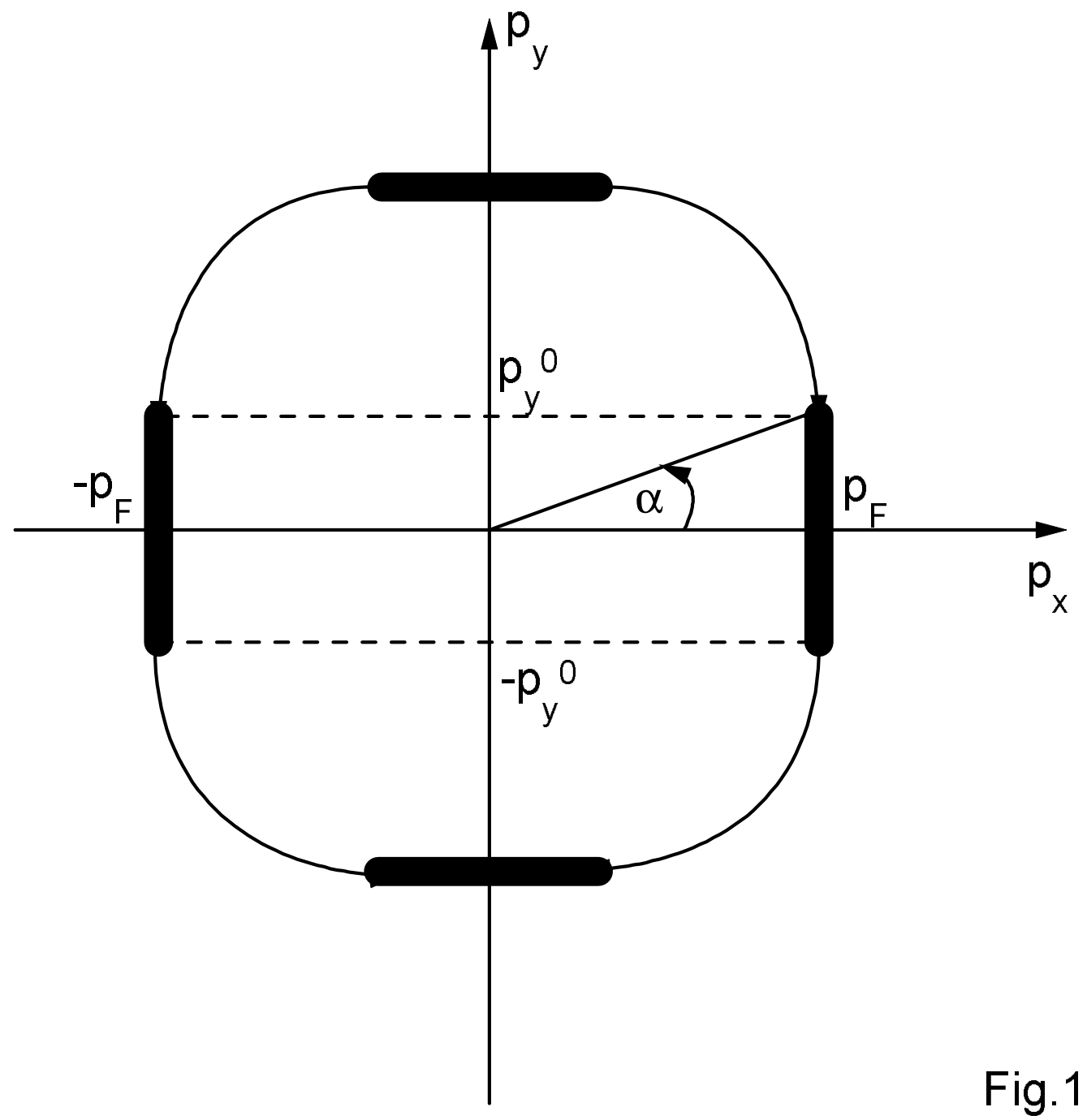



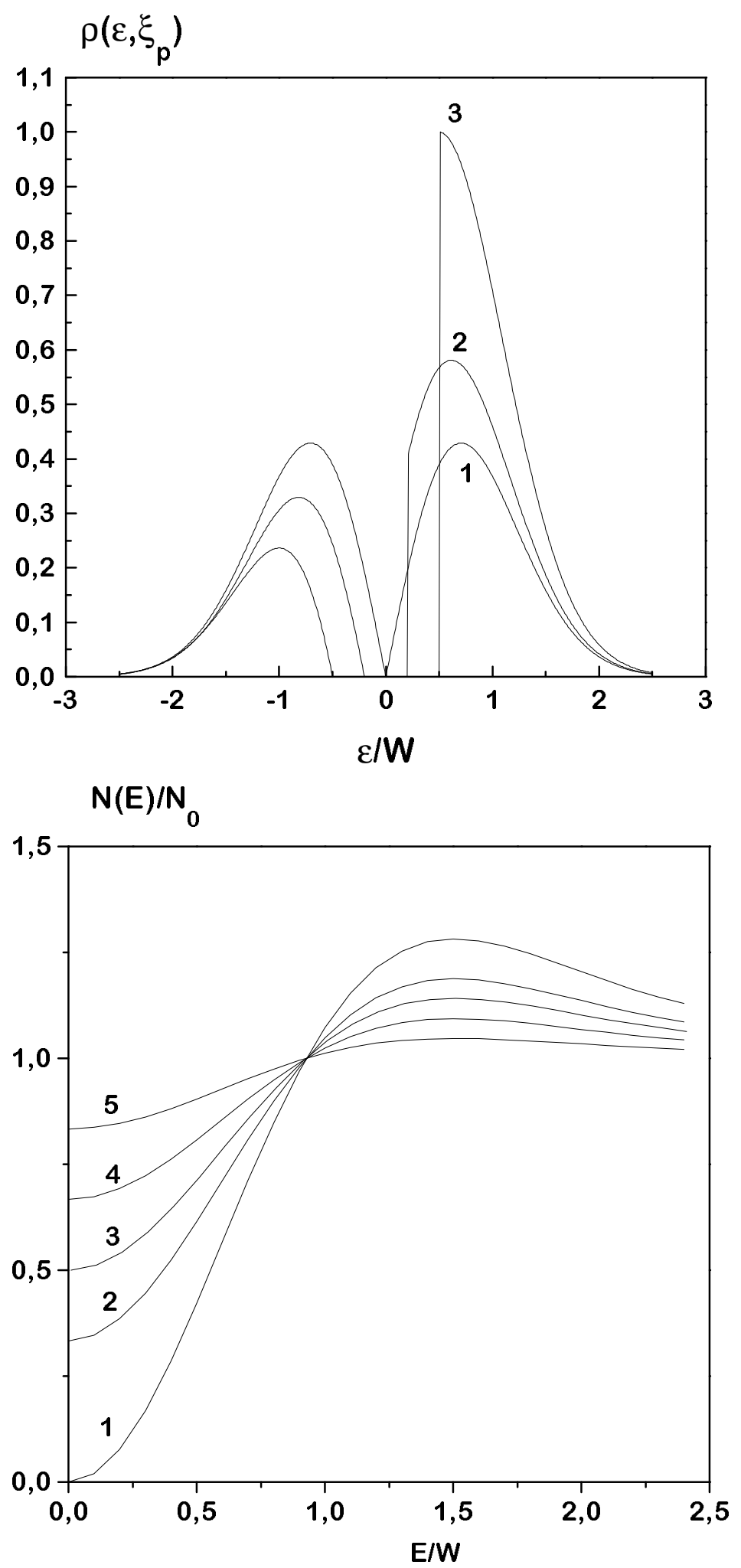

Fig. 2

Fig.3 


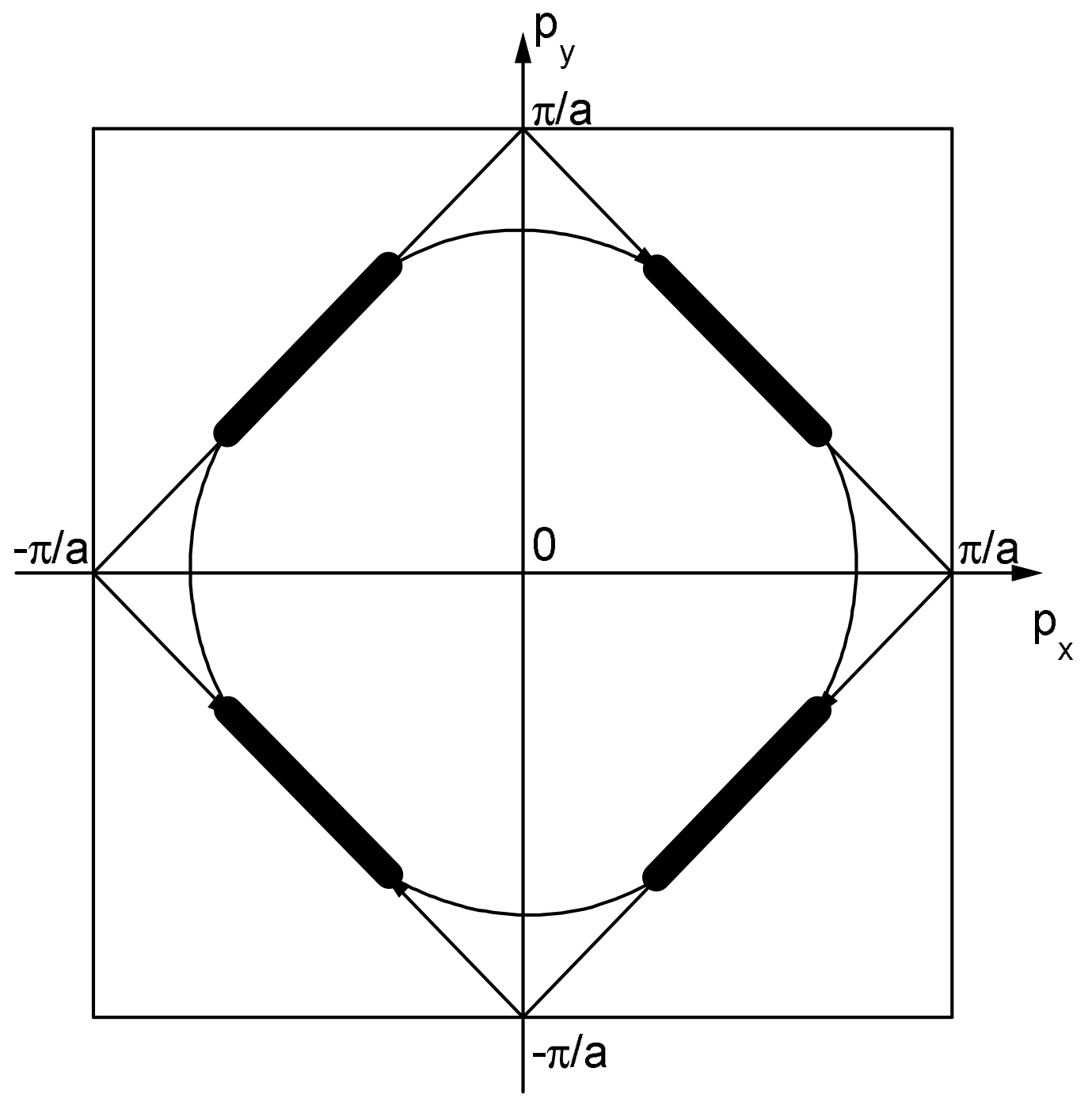

Fig.4 

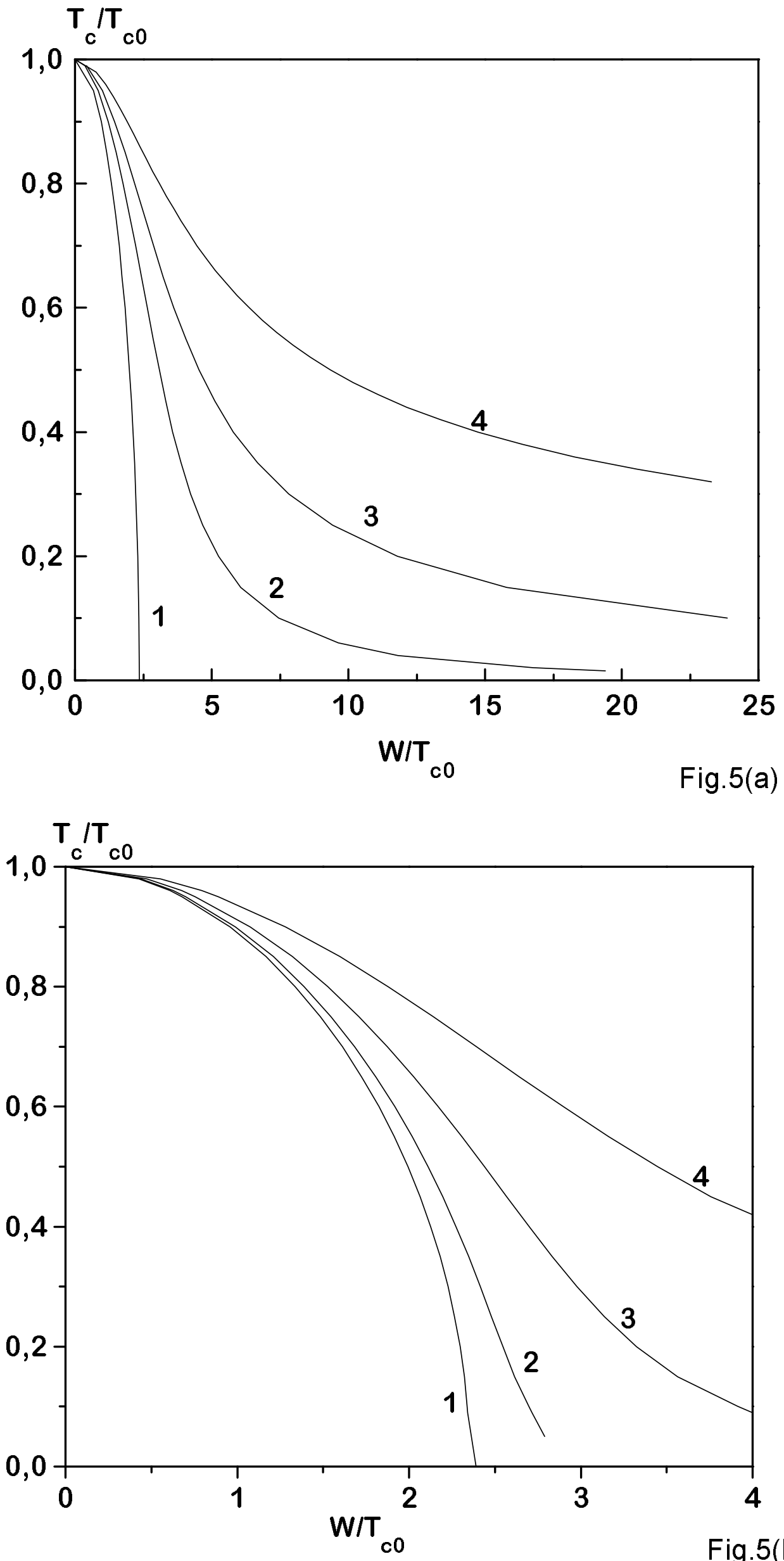

Fig.5(b) 


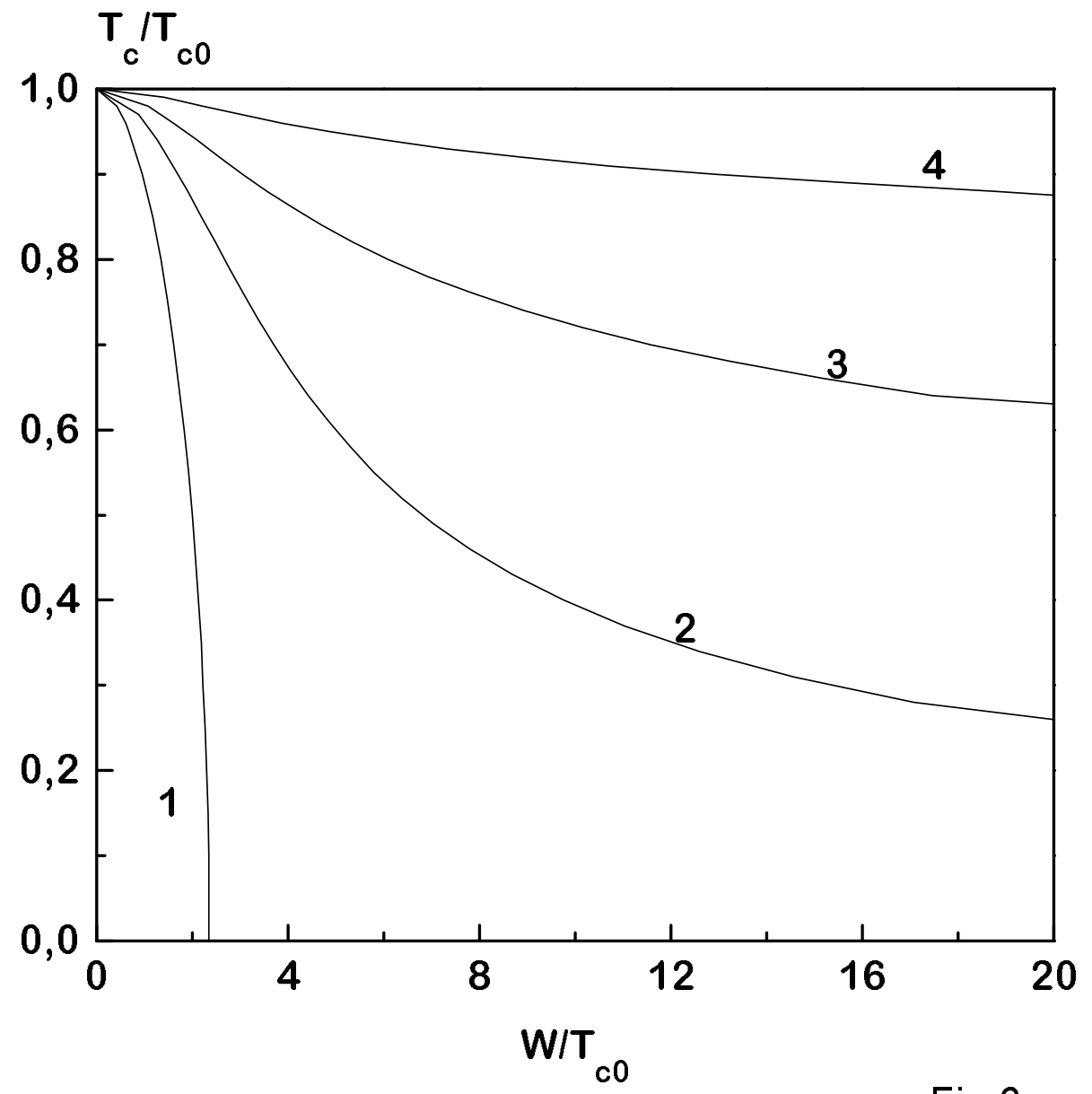

Fig. 6 


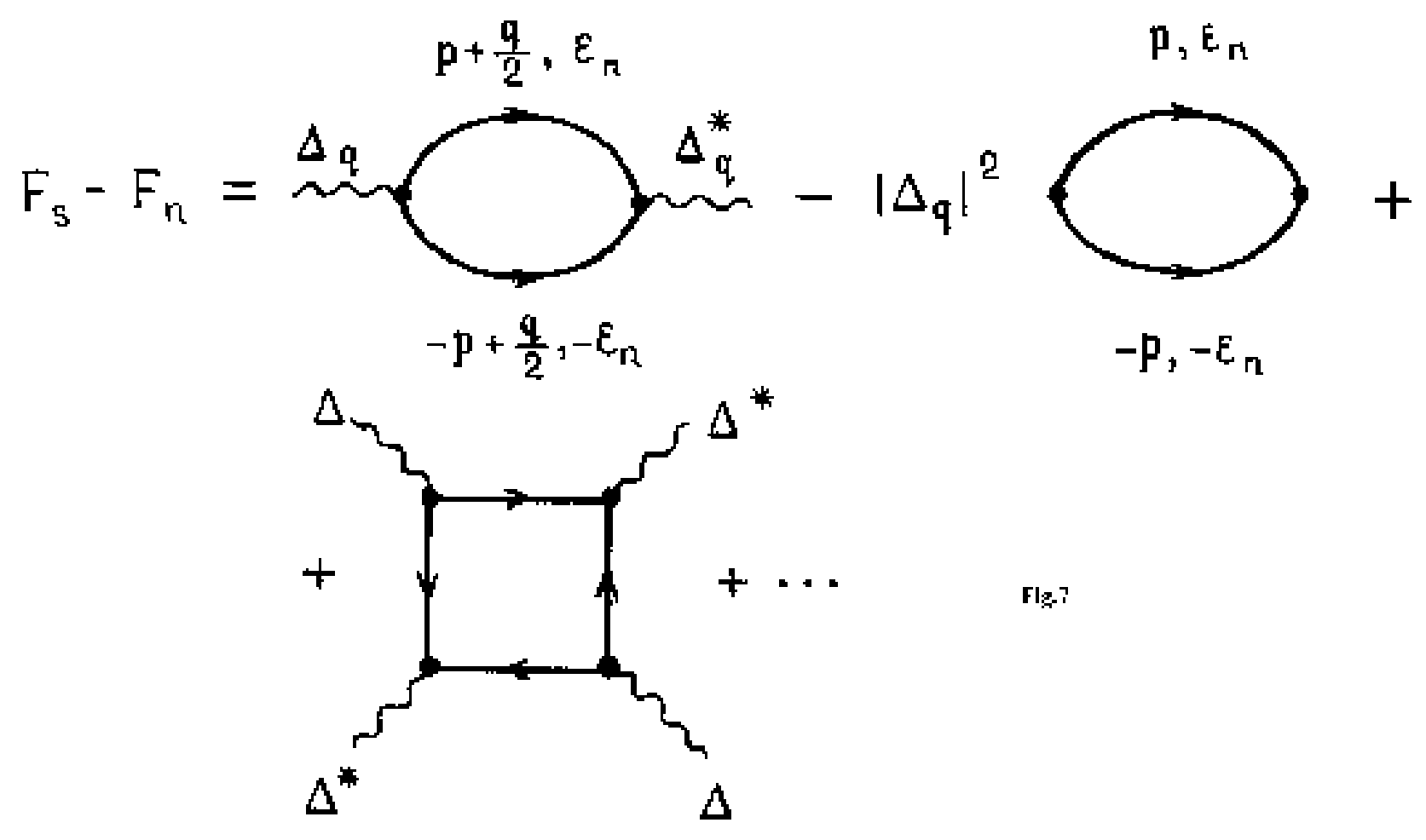




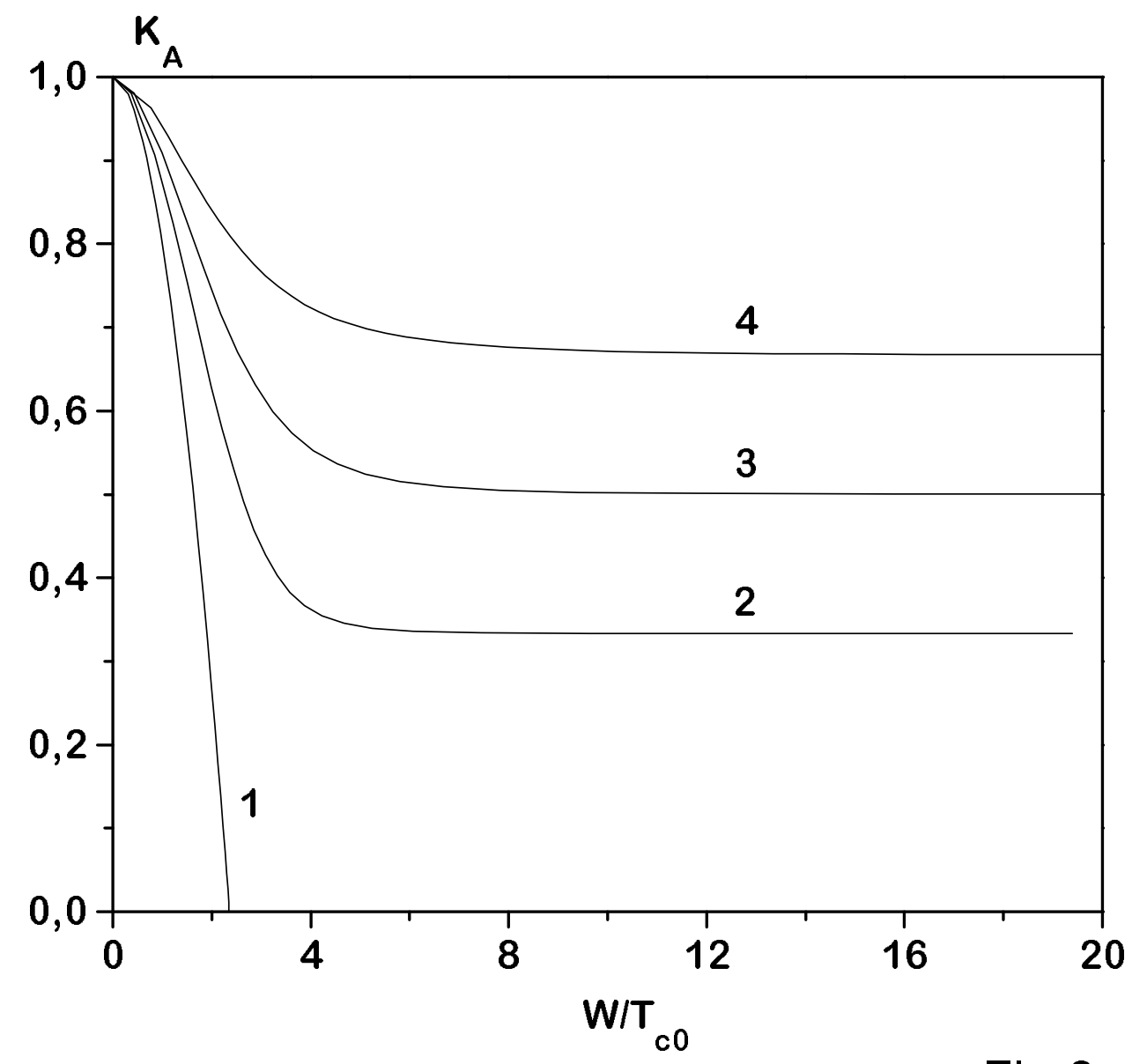

Fig. 8 


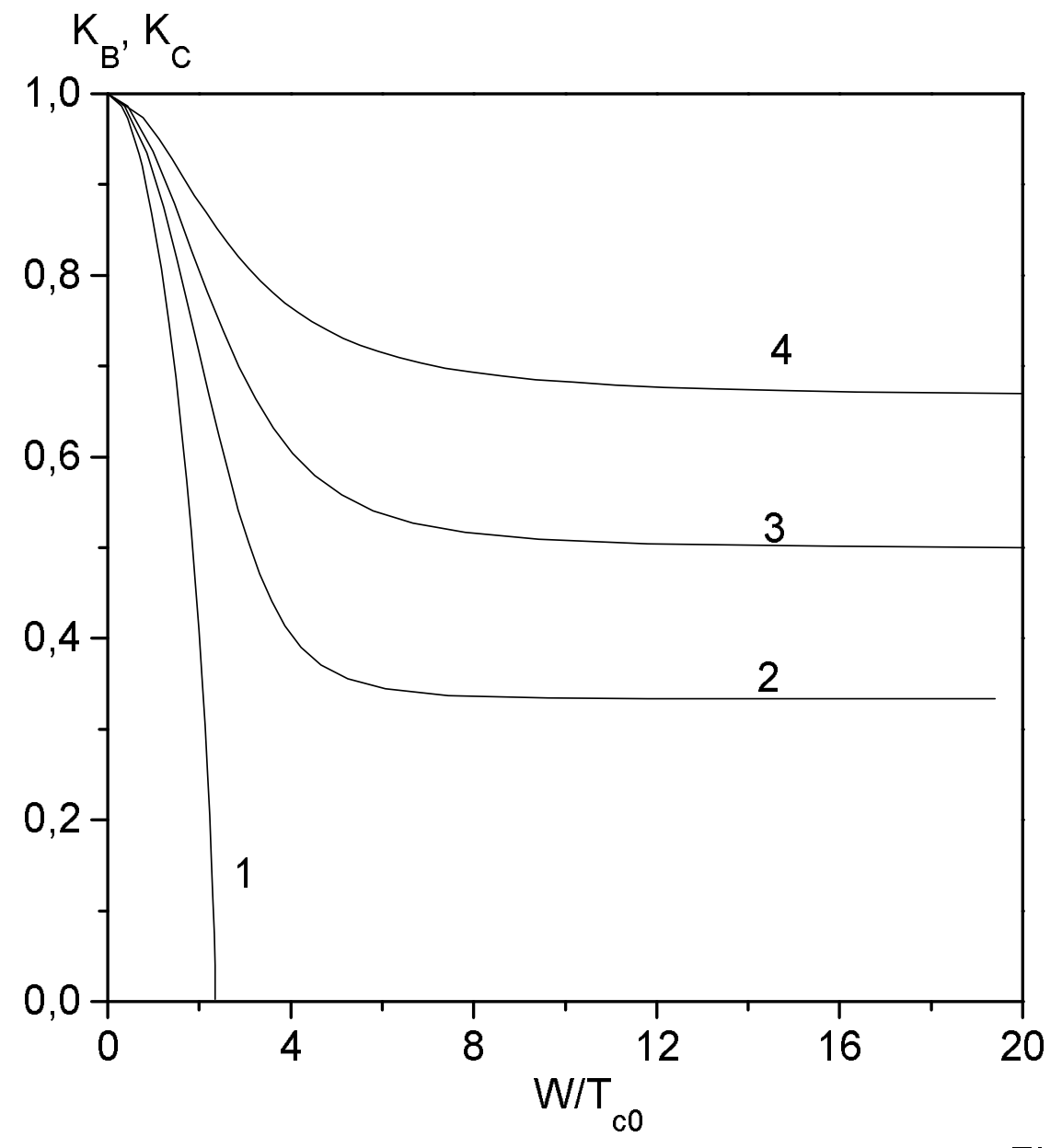

Fig. 9 


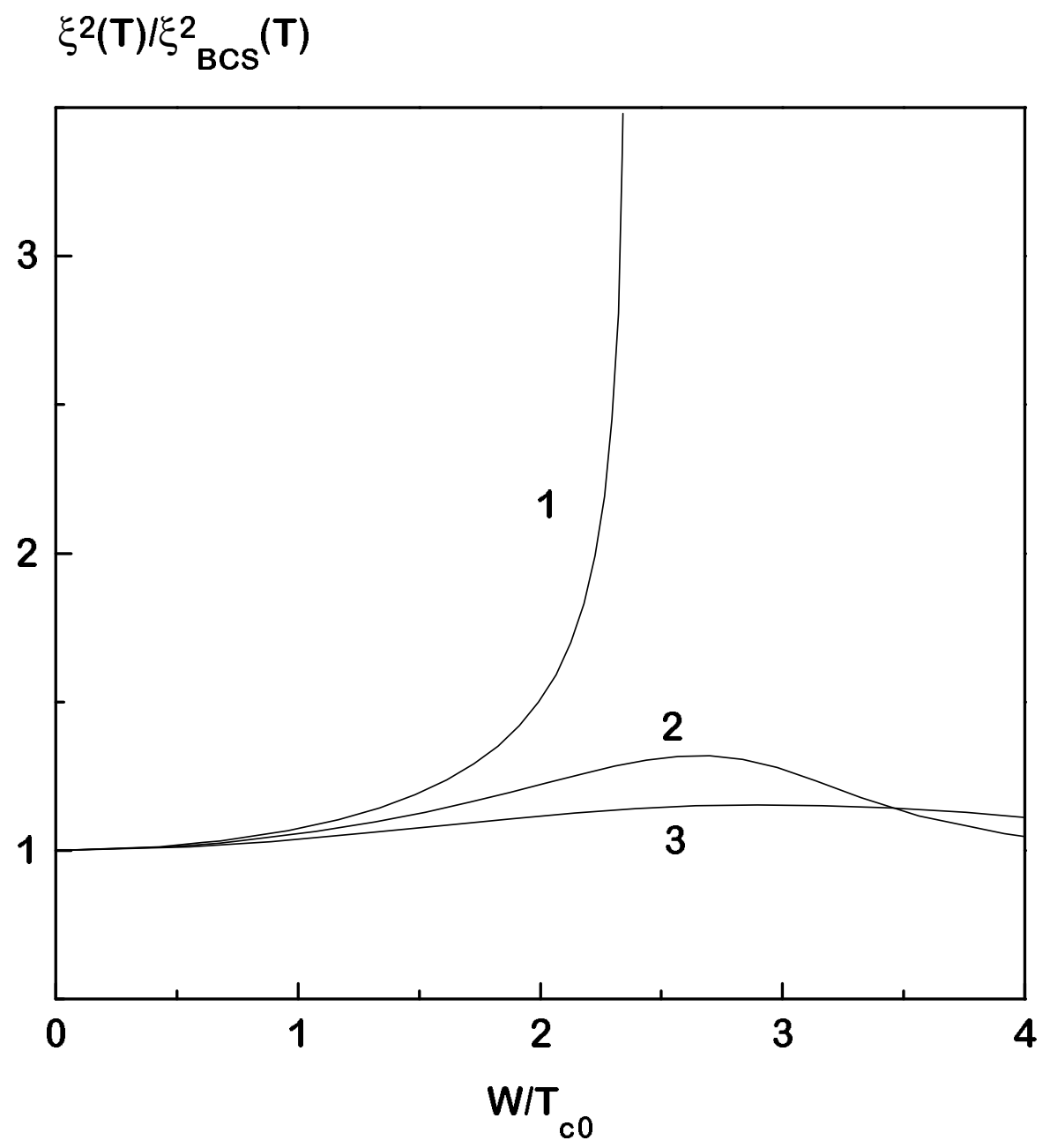

Fig.10 


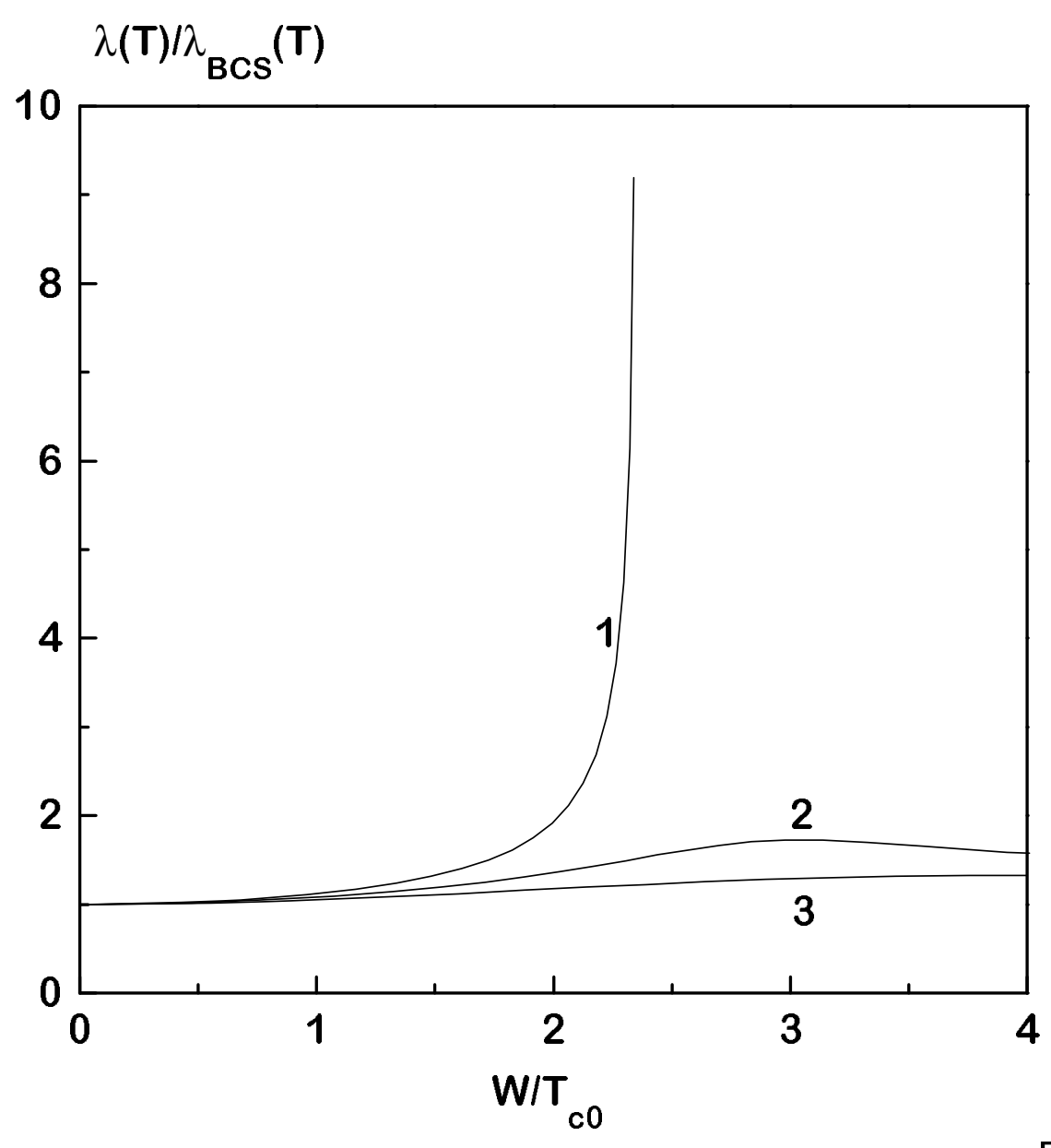

Fig.11 


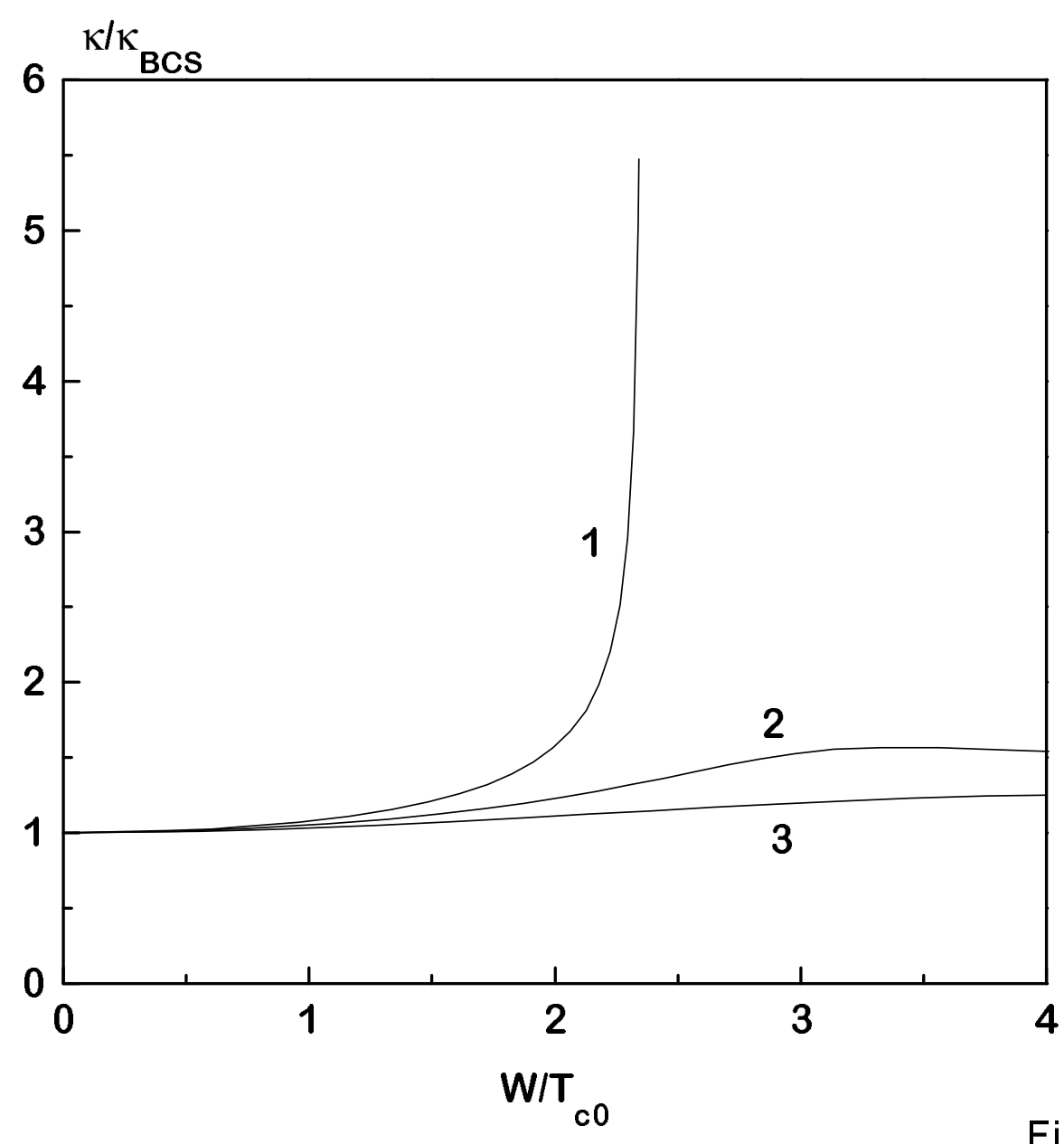

Fig.12 


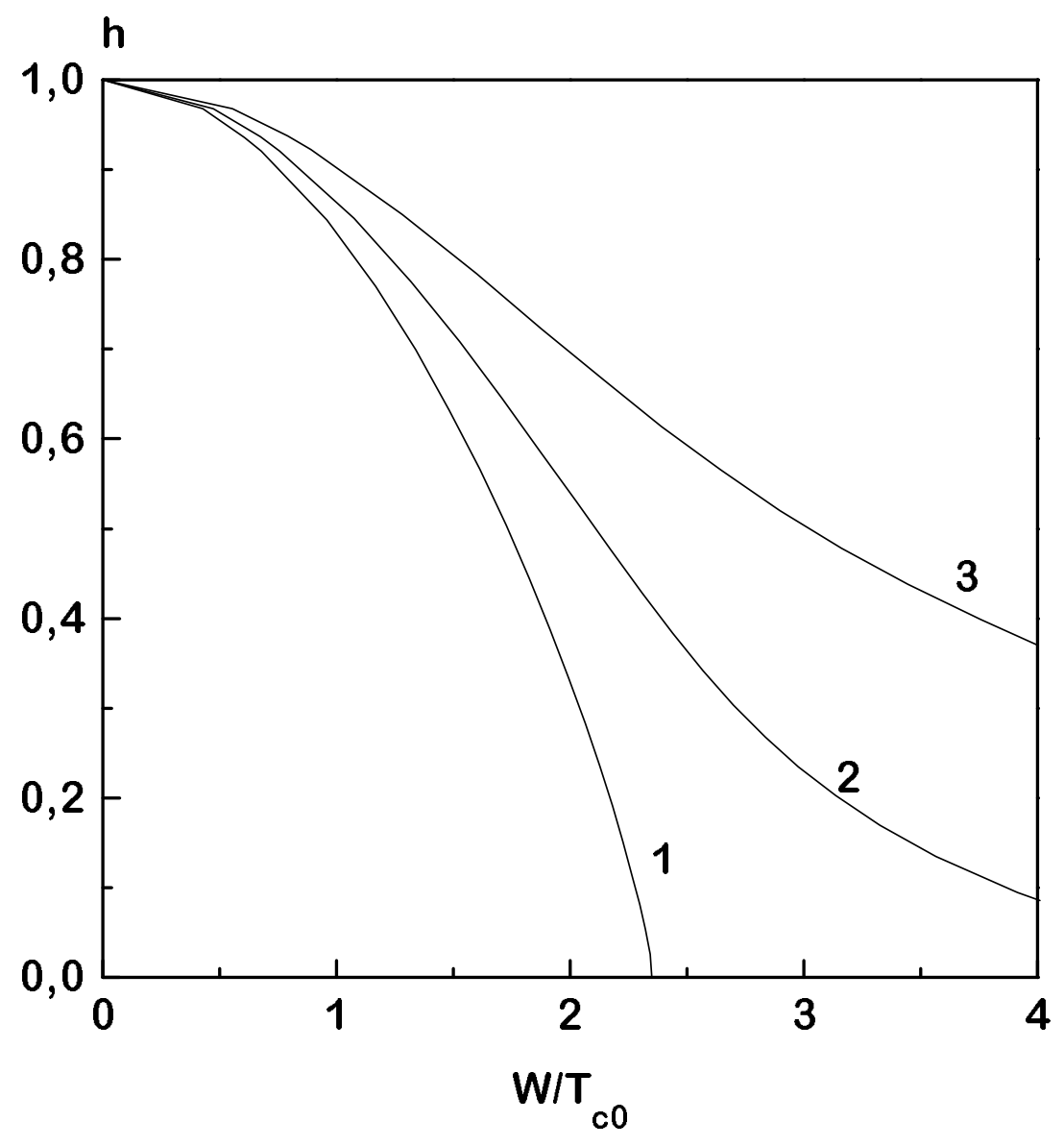

Fig.13 


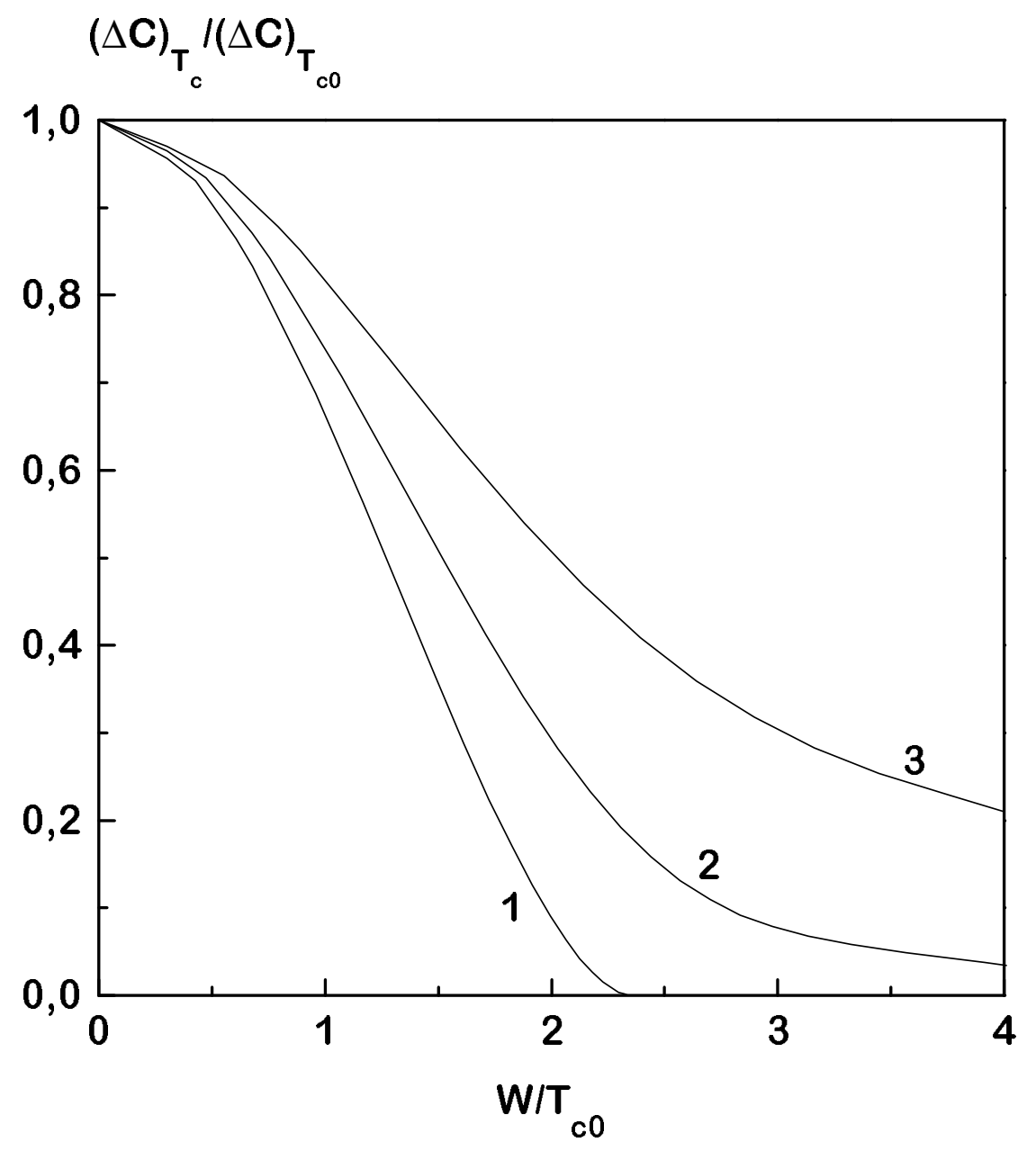

Fig.14 